\title{
High levels of dRYBP induce apoptosis in Drosophila imaginal cells through the activation of reaper and the requirement of trithorax, dredd and $d F A D D$
}

\author{
Inma González ${ }^{1}$, Ana Busturia ${ }^{1}$ \\ ${ }^{1}$ Centro de Biología Molecular "Severo Ochoa” CSIC-UAM, c) Nicolás Cabrera 1, Universidad Autónoma de Madrid, Campus de \\ Cantoblanco, 28049 Madrid, Spain
}

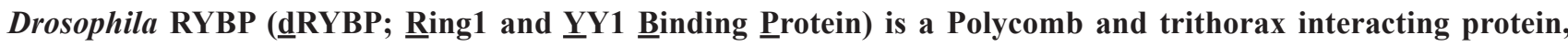
whose homologous RYBP/DEDAF mammalian counterparts exhibit tumor cell-specific killing activity. Here we show that although endogenous dRYBP is not involved in developmental apoptosis, high levels of exogenous dRYBP induce apoptosis in all the imaginal dises of the fly, indicating that dRYBP apoptotic activity is not specific to tumor cells. We also show that dRYBP-induced apoptosis is inhibited by high levels of either p35 or DIAP1 (Drosophila Inhibitor of Apoptosis Protein 1), and requires the function of the pro-apoptotic REAPER, HID and GRIM proteins, the apical caspase DREDD, the adaptor dFADD protein as well as TRITHORAX (TRX), an epigenetic transcriptional regulator. Furthermore, we demonstrate that overexpression of TRX also induces apoptosis in the imaginal discs. Finally, we show that the expression of reaper-lac $Z$ is upregulated both upon dRYBP-induced apoptosis and upon TRXinduced apoptosis in imaginal dises and that the reaper gene is a direct target of dRYBP in Drosophila embryos. Our results indicate that dRYBP triggers in a receptor-mediated apoptotic pathway that also includes TRX-dependent epigenetic regulation of gene expression.

Keywords: dRYBP, apoptosis, trithorax, Polycomb, Drosophila

Cell Research (2009) 19:747-757. doi: 10.1038/cr.2009.29; published online 3 March 2009

\section{Introduction}

Apoptosis affects many aspects of development, including shaping of body structures, controlling cell number and eliminating abnormal, misplaced or harmful cells $[1,2]$. Moreover, cell killing in response to death signal stimuli is essential to remove cells with neoplastic transformation potential $[3,4]$. The pathways controlling apoptosis are highly conserved from flies to humans. The core of the apoptosis cell death machine consists of members of the caspase family of proteases [5] whose activation is regulated either by an extrinsic or by an intrinsic signaling pathway. In Drosophila, the intrinsic pathway is stimulated by death-inducing signals that

Correspondence: Ana Busturia

Tel: +34-91-196-4689; Fax: +34-91-196-4420

E-mail: abusturia@cbm.uam.es

Received 20 November 2008; revised 2 December 2008; accepted 22 December 2009; published online 3 March 2009 activate the RHG family (reaper, hid, grim, sickle and jafra-2) of pro-apoptotic proteins, leading to the activation of the effector caspases and the induction of apoptosis (reviewed in Domingos et al. [6]). Likewise, in mammals the intrinsic pathway is stimulated by intracellular stimuli such as DNA damage and cytotoxic drugs that act inside the cell activating the pro-apoptotic proteins and leading to the activation of caspases [7]. The extrinsic pathway in mammals, also referred to as the receptormediated apoptotic pathway, plays an important role in both development and disease [7]. After an apoptotic stimulus, the TNF/TNFR (tumor necrotic factor/tumor necrotic factor receptor) signaling is activated and mediates cell death through both the activation of the JNK pathway and the activation of caspases. Additionally, in mammals, initiation of receptor-mediated apoptosis activates the formation of the death receptor complex, composed of TNFR associated factors (TRAFs), which function with the adaptor protein FADD to recruit and aggregate procaspase- 8 , thereby activating caspase- 8 through 
mutual cleavage and initiating apoptosis [8]. This pathway, however, is poorly understood in Drosophila. The Drosophila TNF/TNFR homologs are EIGER/WENGEN $[9,10]$. Three Drosophila members of the TRAF family have been identified $[11,12]$. The Drosophila homologs of the DED (Death Domain)-containing protein FADD and caspase- 8 are dFADD and DREDD, respectively [13, 14]. However, unlike its mammalian counterpart, the apoptotic effect of EIGER requires neither the activity of the caspase- 8 homolog DREDD nor the adaptor dFADD protein, but is completely dependent on the activation of the JNK signaling pathway [10].

The $d R Y B P$ gene encodes a phylogenetically conserved 150-amino acid protein with a conserved amino terminus, which contains a NZF ( $\underline{N} 14$ zinc finger) type zinc-finger [15] (Figure 1L) and a carboxy-terminus with so far no identified functional domains. Recently, a subgroup of NZF domains that includes that of RYBP has been shown to possess ubiquitin binding activity [16]. We have previously shown that loss of $d R Y B P$ function produces phenotypes that are highly variable in both penetrance and expressivity [17]. Moreover, we showed that dRYBP interacts genetically and molecularly with Polycomb (PcG) and trithorax (trxG) proteins and that its overexpression generates a small wing phenotype $[17$, 18].

In mammals, high levels of the RYBP protein have been found to have tumor-specific killing activity, a characteristic that could potentially be exploited to develop anti-cancer treatments [19]. Furthermore, the RYBP protein has been found to interact with Apoptin, a protein known to be active in killing tumor cells [20]. As induction of apoptosis is a common mechanism underlying cancer therapy, understanding the mechanisms controlling tumor cell-specific killing may facilitate the development of improved therapies.

We show here that overexpression of dRYBP and overexpression of TRITHORAX, an epigenetic regulator of transcription [21], induce apoptosis in the imaginal discs. Moreover, we show that high levels of dRYBP and high levels of TRITHORAX activate the expression of a reaper-lac $Z$ transgene. We also show that the pro-apoptotic gene reaper is a direct target of the dRYBP protein and that dRYBP-induced apoptosis requires the function of reaper and trithorax. These findings reveal the participation of a factor known to be an epigenetic regulator of gene transcription in the control of apoptosis. Furthermore, we show that dRYBP-induced apoptosis is independent of the JNK pathway but requires DREDD and AFADD, two factors previously shown not to be associated with receptor-mediated apoptosis in Drosophila [10].

\section{Results}

\section{Endogenous dRYBP is not required for developmental} apoptosis

Loss of function of the $d R Y B P$ gene results in phenotypes that are highly variable both in penetrance and in expressivity [17]. In this report, we have investigated whether the inactivation of dRYBP function using $\mathrm{RNAi}_{\mathrm{dRYBP}}$ transgenic lines and loss of function $d R Y B P$ mutations have an effect on apoptosis. We first performed caspase-3 [22] and Terminal deoxynucleotidyl transferase-mediated dUTPNick End Labelling (TUNEL) staining in the wing imaginal discs of $s d-G A L 4 /+$; UAS$R N A i_{d R Y B P} / U A S-R N A i_{d R Y B P}$ larvae and the wing imaginal discs of $d R Y B P^{l} / d R Y B P^{l}$ larvae and found similar results to those in the wild-type wing imaginal discs. Second, we looked at Acridine Orange staining during embryonic development of the progeny of $d R Y B P^{l} / C y O-G F P$ stock. Compared to wild-type embryos $\left(D f(1)^{\text {w67c23 }}\right.$, see Materials and Methods), mutant (non-GFP) embryos displayed no appreciable changes in cell death throughout the central nervous system during nerve cord condensation in stage 16 embryos or in the embryonic head (data not shown). These results suggest that $d R Y B P$ function is not required for developmental apoptosis.

\section{Exogenous dRYBP expression induces apoptotic cell death}

Using the GAL4/UAS system, we observed that overexpression of the dRYBP protein in wing imaginal discs produces small adult wings $[17,18]$. We asked whether the reduction of wing size was due to the induction of apoptosis. We have used several GAL4 drivers (en-Gal4, ci-Gal4, sd-Gal4, nub-Gal4, 248-Gal4 and dll-Gal4; see Materials and Methods) to induce high levels of dRYBP and found that overexpression of dRYBP induces apoptosis in all imaginal discs as evidenced by the activation of caspase-3 (Figure 1D-1K). We have also investigated whether the murine RYBP/DEDAF protein can induce apoptosis in wing imaginal discs. Similar to the expression of the Drosophila protein (Figure 1D), we found that the wing imaginal discs of $s d-G a l 4 /+$; UAS-RYBP murine/+ show induction of apoptosis (not shown). To assess the contribution of apoptosis to the dRYBP overexpression phenotype, we used the baculovirus caspase inhibitor p35 to block apoptosis [23]. Co-expression of p35 and dRYBP in nub-Gal4/UAS dRYBP; UAS p35/+ represses the dRYBP-induced apoptosis (Figure 1I, $1 \mathrm{M}$ and $1 \mathrm{~N}$ ). Moreover, the small-size wing phenotype observed when high levels of dRYBP are expressed in the wing is rescued when p35 is also overexpressed (data not shown). This result indicates that caspases are necessary 

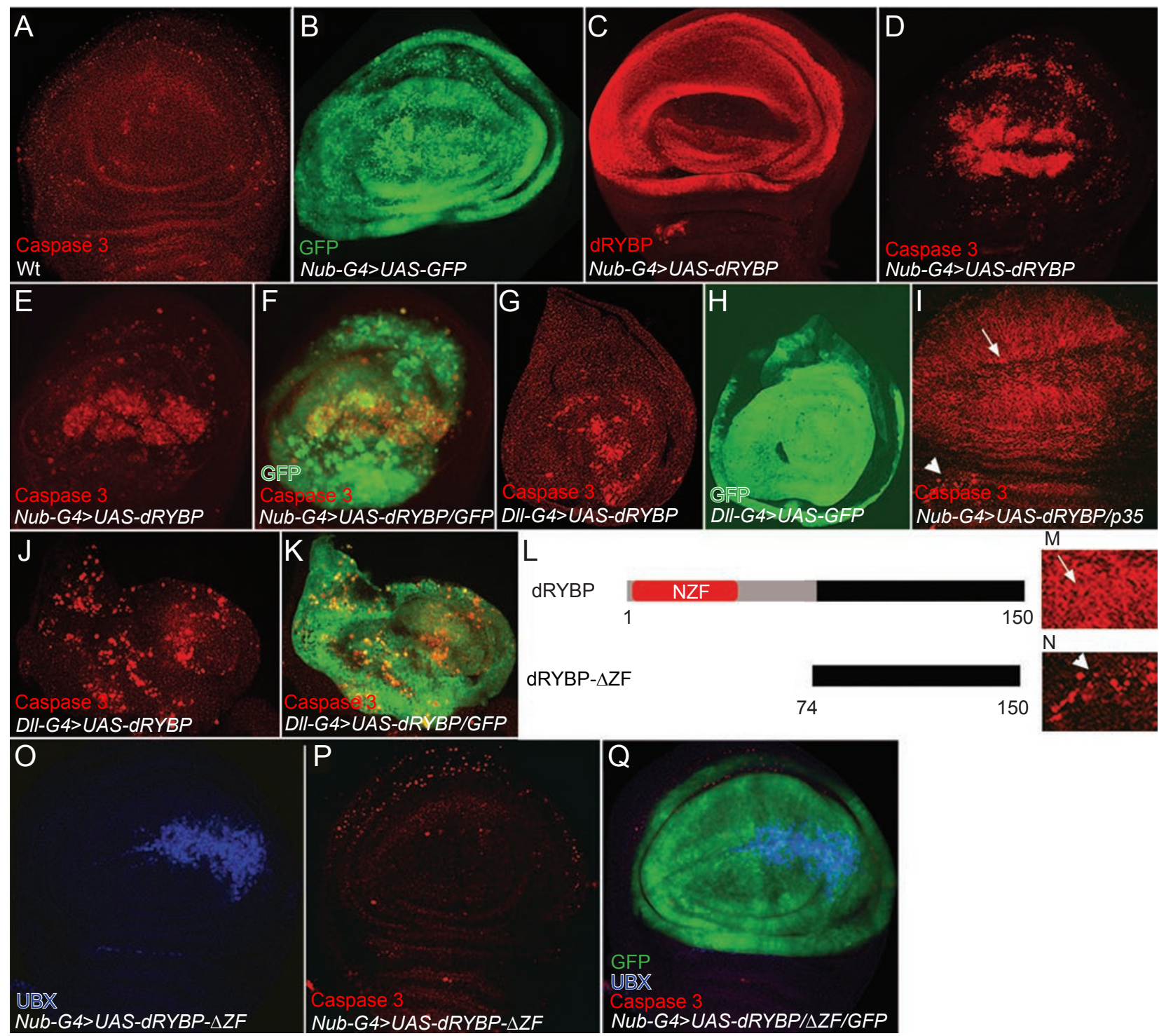

UBX
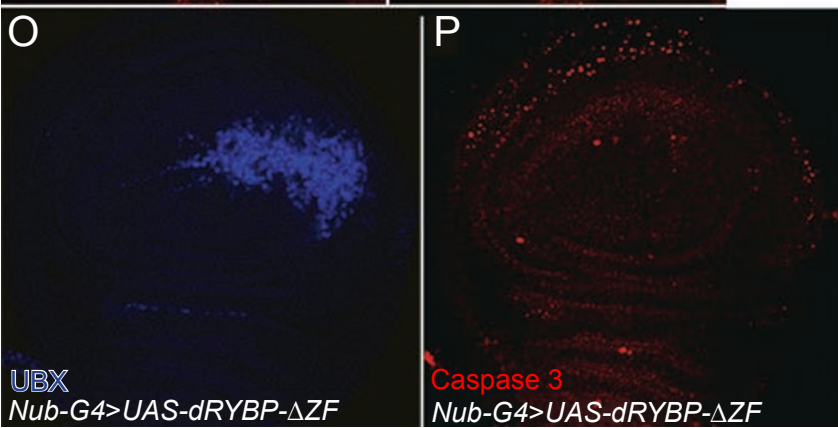

Figure 1 Exogenous dRYBP expression induces apoptotic cell death. (A) Activated caspase-3 (C3) staining (red) in a wildtype wing disc. (B) GFP expression (green) in a nub-Gal4, UAS-GFP wing disc showing the nub-Gal4 line domain of expression in the wing disc. (C) dRYBP expression (red) in a nub-Gal4, UAS-GFP/ UAS-dRYBP wing disc. (D) C3 staining (red) in a nub-Gal4, UAS-GFP/UAS-dRYBP wing disc. (E) C3 staining (red) in a nub-Gal4, UAS-GFP/UAS-dRYBP haltere disc. (F) Merged expression of GFP (green, showing the domain of expression of the nub-Gal4 line in the haltere disc) and C3 staining (red) in a nub-Gal4, UAS-GFP/UAS-dRYBP haltere disc. (G) C3 staining (red) in a dll-Gal4, UAS-GFP/UAS-dRYBP third leg disc. (H) GFP expression (green) in a dII-Gal4, UAS-GFP/UAS-dRYBP third leg disc, showing the dll-Gal4 line domain of expression in the leg disc. (I) C3 staining (red) in a nub-Gal4, UAS-GFP/UAS-dRYBP/+; UAS p35/+ wing disc. This wing disc illustrates the differential staining of the anti-C3 antibody when activated caspases are inhibited by overexpression of p35 in the nub-Gal4 domain (arrow, see detail in $\mathbf{M}$ ) and when activated caspases are not inhibited by overexpression of $p 35$ outside the nub-Gal4 domain (arrowhead, see detail in N). (J) C3 staining (red) in dII-Gal4, UAS-GFP/UAS-dRYBP antenna disc. (K) Merged expression of GFP (green) and C3 staining (red) in dll-Gal4, UAS-GFP/UAS-dRYBP antenna disc. (L) Scheme of the UAS-dRYBP and UAS-dRYBP- $\triangle Z F$ constructs. The NZF (indicated in red) is located at the amino-terminus of the protein. (M) Detail of I - arrow showing staining of C3 in p35-apoptosis inactivated cells. (N) Detail of I - arrowhead showing staining of C3 in apoptotic cells. (O) UBX expression (blue) in nub-Gal4, UAS-GFP/dRYBP- $\triangle Z F$ wing disc. (P) C3 staining in a nubGal4, UAS-GFP/dRYBP- $\triangle Z F$ wing disc. (Q) Merged expression of UBX, C3 and GFP in nub-Gal4, UAS-GFP/dRYBP- $\triangle Z F$. The de-repression of UBX only takes place in a few cells within the nub-Gal4 domain. 
for dRYBP-induced apoptosis.

We have shown that overexpression of dRYBP in wing discs causes the homeotic Ultrabithorax (UBX) protein [24] to be expressed in a limited number of wing disc cells $[17,18]$. This UBX expression is not dependent on the presence of the NZF domain of the dRYBP protein as overexpression of the dRYBP- $\triangle \mathrm{ZF}$ and fulllength proteins (Figure 1L) produced the same pattern of UBX expression in wing discs (Figure 1O). Furthermore, in contrast to overexpression of full-length dRYBP, wing discs of $n u b-G a l 4 / U A S-d R Y B P-\triangle Z F$ larvae do not show activation of caspase-3 (Figure 1P). As shown in Figure 1 , the wing imaginal discs overexpressing the truncated dRYBP- $\triangle$ ZF protein show expression of the homeotic UBX protein (Figure 1O) but do not show activation of caspase-3 (Figure 1P), indicating that the induction of apoptosis is not a consequence of UBX expression.

These results demonstrate that exogenous expression of either dRYBP or murine RYBP induces apoptosis in the Drosophila imaginal disc cells. Moreover, this induction is not a consequence of UBX expression. Finally, this induction of apoptosis does not require the cells to be tumoral as it occurs in normal imaginal disc cells.

dRYBP-induced apoptosis is dependent on both proapoptotic and anti-apoptotic factors

To gain insight into the molecular pathways controlling dRYBP-induced apoptosis, we investigated the requirement for pro-apoptotic factors [25] which act by inhibiting the activity of the Drosophila Inhibitor of Apoptosis (DIAP) anti-apoptotic proteins [26]. Three of the pro-apoptotic genes described in Drosophila - reaper, hid and grim - are contained within a single genomic deficiency - Df (3L) H99 ([27]; see Materials and Methods). Imaginal discs from $n u b-G a l 4 / U A S d R Y B P ; D f$ (3L) H99/+ larvae show a marked reduction in dRYBPinduced apoptosis (Figure 2B) and a partial rescue of the wing size (data not shown). Furthermore, high levels of the DIAP1 protein in $n u b-G a l 4 / U A S$ dRYBP; UAS $D I A P 1 /+$ larvae led to a complete inhibition of apoptosis in wing imaginal discs (Figure 2C) and accordingly, rescue of the wing size (data not shown). Together, these results show that $\mathrm{dRYBP}$-induced apoptosis requires the function of the pro-apoptotic Reaper, Hid and Grim proteins, and that this process is inhibited by high levels of DIAP1. This, in turn, suggests that dRYBP-induced apoptosis is dependent on the canonical apoptotic pathways in which these factors function.

The reaper gene promoter is a direct target of the $d R Y B P$ protein

To investigate whether dRYBP regulates the expres- sion of apoptotic genes, we studied the effect of dRYBP on the expression of reaper by monitoring $\beta$-gal expression in $s d-G a l 4 /+$; UAS-dRYBP/rpr-lacZ wing imaginal discs. As shown in Figure 2G-2I, expression of rpr-lacZ in the wing discs is activated by dRYBP overexpression, indicating that high levels of dRYBP cause mis-regulation of reaper expression.

We next studied whether the reaper gene is bound by the dRYBP protein by performing chromatin immunoprecipitation (ChIP) experiments in wild-type Drosophila embryos using an antibody against dRYBP. Quantitative PCR was used to identify regions in and around the reaper gene enriched in dRYBP ChIP products and that are, therefore, likely to be bound by the dRYBP protein (Materials and Methods). As shown in Figure 2J, the reaper gene promoter is enriched in the dRYBP ChIP products compared to the control region.

These results clearly indicate that in wild-type imaginal discs the pro-apoptotic gene reaper is a direct target of dRYBP and suggest that its transcription is activated when $\mathrm{dRYBP}$ is expressed at high levels.

dRYBP-induced apoptosis is independent of the JNK pathway and is dependent on the DED-domain containing proteins $A F A D D$ and DREDD

Activation of the receptor-mediated extrinsic death pathway upon apoptotic stimuli induces cell death through activation of the JNK pathway [9, 10]. In Drosophila, activation of the JNK pathway is independent of the DED-domain containing proteins DREDD (caspase- 8 in humans) and dFADD (the Fas-associated death-domain containing protein, FADD in humans) [10]. In humans, however, receptor-mediated apoptosis is dependent on FADD and caspase-8 [28]. By monitoring puckered expression using puckered-lac ${ }^{E 69}$ transgenic flies [29], we studied whether dRYBP-induced apoptosis is able to activate the JNK pathway. We found that the $\beta$ gal expression pattern is identical in wing imaginal discs from $s d-G a l 4 /+$; UAS-dRYBP/+; puc-lacZ/+ and from control puc-lacZ/+ larvae, indicating that exogenous dRYBP does not activate the JNK pathway (not shown).

We next studied the requirement of DREDD and dFADD in dRYBP-induced apoptosis. $d r e d d^{D 44} /$ dred $^{D 44}$; $n u b-G a l 4 / U A S-d R Y B P$ wing imaginal discs do not show activation of caspase-3 (Figure 2D). Additionally, nubGal4/UAS-RNAi ${ }_{\mathrm{FADD}}{ }^{\mathrm{M} 8}$; UAS-dRYBP/+ wing imaginal discs show no activation of apoptosis (Figure 2E). The overexpression of dRYBP in the absence of either dredd or $d F A D D$ results in flies with wings of a bigger size (data not shown) due to the absence of apoptosis in those mutant conditions. These results indicate that, like receptormediated apoptosis in mammals, exogenous dRYBP- 

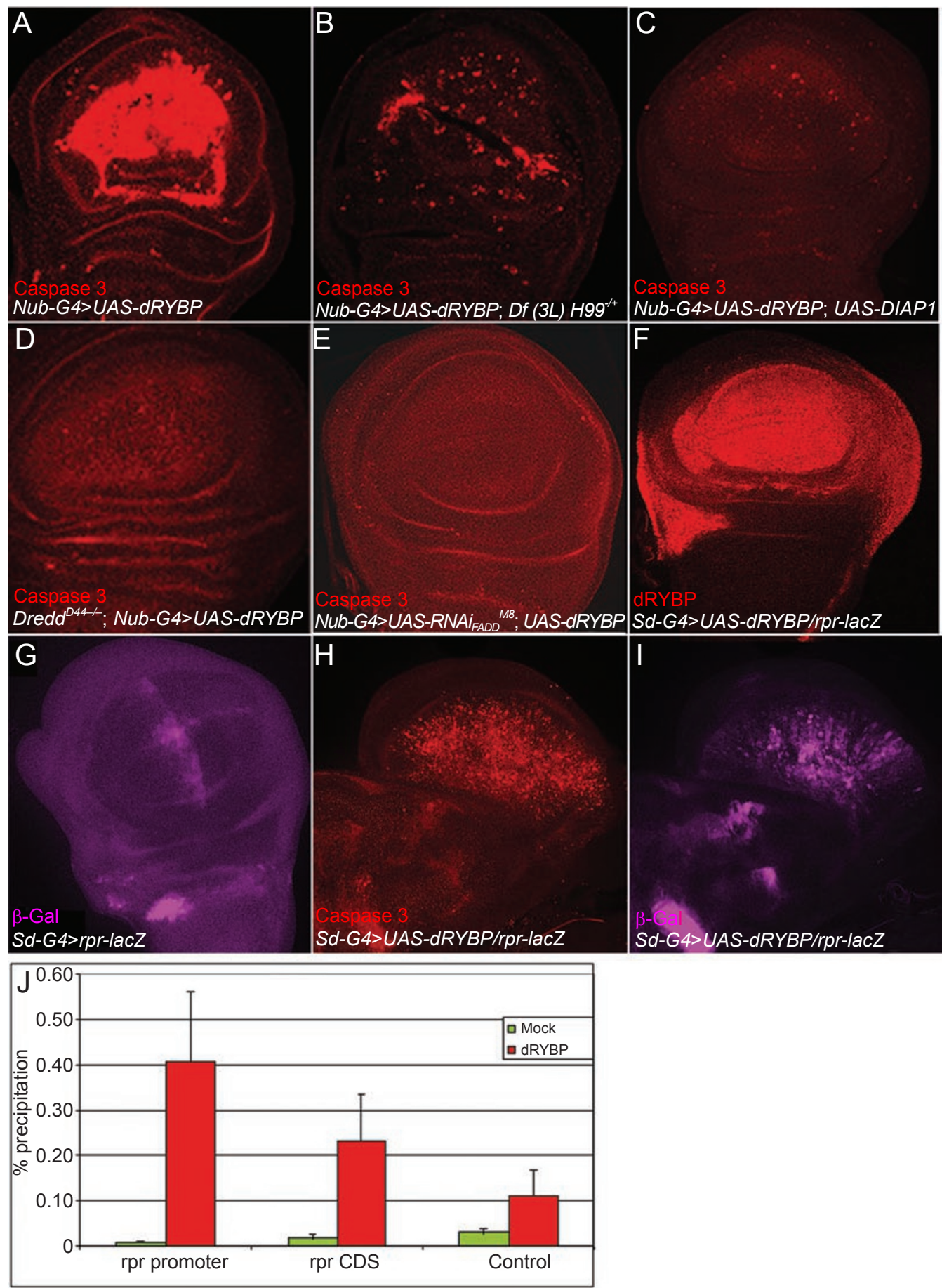

Figure 2 Factors involved in the dRYBP induction of apoptosis. (A) C3 staining (red) in nub-Gal4, UAS-GFP/dRYBP wing disc. (B) C3 staining (red) in nub-Gal4, UAS-GFP/dRYBP; Df(3L)H99/+ wing disc. Apoptosis is diminished. (C) C3 staining (red) in nub-Gal4, UAS-GFP/dRYBP; UAS-DIAP1/+. Apoptosis is almost absent. (D) C3 staining (red) in $d r e d d^{D 44} / d r e d d^{D 44}$; nubGal4, UAS-GFP/UAS-dRYBP wing imaginal disc. Apoptosis is greatly suppressed. (E) C3 staining (red) in nub-Gal4, UASGFP/UAS-RNAi $i_{F A D D} M 8$; UAS-dRYBP/+ wing disc. Apoptosis is nearly completely absent. (F) dRYBP expression (red) in a sdGal4/+; UAS-dRYBP/rpr-lacZ wing disc indicating the domain of expression driven by the sd-Gal4 line. (G) $\beta$-Gal expression (purple) of a wing disc sd-Gal4/+; rpr-lacZ showing the expression of the reaper-lacZ construct, which is not affected by the presence of the sd-Gal4 line. (H) C3 staining (red) in sd-Gal4/+; UAS-dRYBP/rpr-lacZ wing disc. (I) $\beta$-Gal expression (purple) in a sd-Gal4/+; UAS-dRYBP/rpr-lacZ wing disc. (J) ChIP with dRYBP antibody in 4-12 h embryos. dRYBP antibody (orange bars). Mock (green bars). The immunoprecipitated DNA was analyzed by qPCR using primers for the promoter region of reaper (rpr), for coding sequences (CDS) of reaper and for robo 3 gene as a control. 
induced apoptosis in fly imaginal discs is dependent on DREDD and dFADD expression.

dRYBP-induced apoptosis is trithorax dependent but Polycomb and Sex Comb extra independent

Several lines of evidence indicate that dRYBP interacts with the Polycomb group (PcG) and trithorax group (trxG) of proteins $[17,18]$. We used $\mathrm{PcG}$ and trxG mutant backgrounds to investigate whether apoptosis induced by dRYBP is dependent on these proteins. The activation of caspase- 3 is clearly diminished in wing imaginal discs of $n u b-G a l 4 / U A S$ dRYBP; $\operatorname{trx}^{E 2} /+$ larvae (Figure 3B), while there is no change in either nub-Gal4/

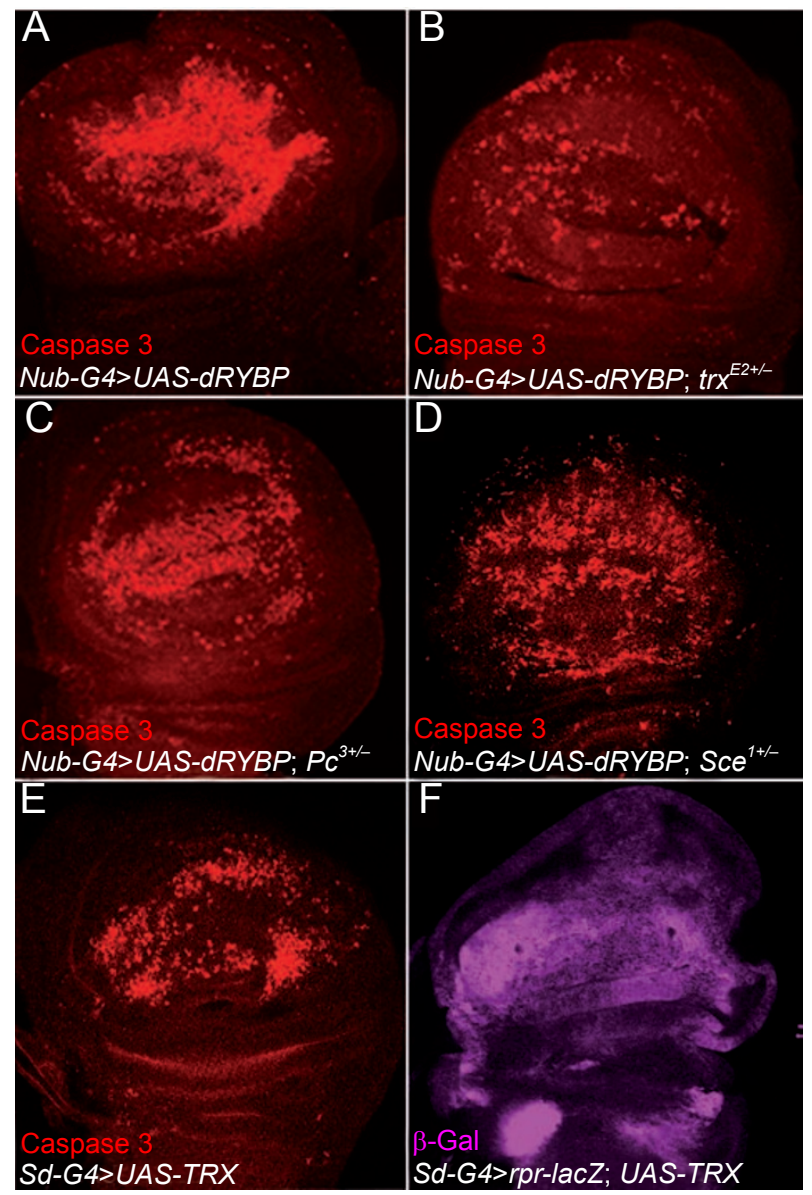

Figure 3 dRYBP-induced apoptosis is trithorax dependent and high levels of Trithorax induce apoptosis. (A) C3 staining (red) in a nub-Gal4, UAS-GFP/UAS-dRYBP wing disc. (B) C3 staining (red) in a nub-Gal4, UAS-GFP/UAS-dRYBP; trx ${ }^{E 2} /+$ wing disc. Note the decrease, compared with (A), in the number of C3 stained cells. (C) C3 staining (red) in a nub-Gal4, UAS-GFP/ $U A S-d R Y B P ; P C^{3} /+$ wing disc. (D) C3 staining (red) in a nubGal4, UAS-GFP/UAS-dRYBP; Sce $1 /+$ wing disc. (E) C3 staining (red) in a sd-Gal4/+; UAS-TRX/+ wing disc. (F) $\beta$-Gal expression (purple) in a sd-Gal4/+; rpr-lacZ/+; UAS-TRX/+ wing disc.
UAS dRYBP; $P c^{3} /+$ larvae (Figure 3C) or $n u b-G a l 4 / U A S$ $d R Y B P ; S_{C e} e^{l}++$ larvae (Figure 3D). These results lead to the conclusion that trithorax, but not Polycomb or Sex comb extra, affects dRYBP-induced apoptosis, although it is possible that lack of only one dose of $P_{c}$ or Sce is not sufficient to influence the induction of apoptosis. These results indicate that the trithorax protein functions in dRYBP-induced apoptosis and links the epigenetic regulation mediated by the trxG genes to the processes of programmed cell death.

Exogenous TRITHORAX induces apoptosis and activation of reaper

We investigated whether developmental apoptosis is modified in $t r x^{E 2}$ homozygous mutant embryos and found that activated caspase- 3 staining in those embryos is similar to wild-type embryos (data not shown), suggesting that the Trithorax protein is not required in developmental apoptosis. We next investigated whether high levels of POLYCOMB, SEX COMB EXTRA and TRITHORAX are capable of inducing apoptosis. Wing imaginal discs from neither nub-Gal4/+; UAS-SCE/+ nor nub-Gal4/+; $U A S-P C /+$ larvae showed induction of apoptosis (data not shown). However, a high level of TRITHORAXinduced apoptosis was indicated by the clear activation of caspase-3 in $s d-G a l 4 /+$; UAS-TRX /+ imaginal discs (Figure 3E). Moreover, to study whether exogenous TRX is capable of inducing the expression of pro-apoptotic genes, we looked at the $\beta$-gal expression in imaginal discs of $s d-G a l 4 /+$; rpr-lacZ/+; UAS-TRX/+ larvae and found that $r p r-l a c Z$ expression is activated in the wing imaginal discs (Figure 3F). These results demonstrate the ability of exogenously expressed trithorax protein to both induce apoptosis as well as activate the expression of the pro-apoptotic reaper gene.

\section{Discussion}

\section{High levels of exogenous dRYBP induce apoptosis}

In the present work, we have shown that loss of function of $d R Y B P$ does not affect the apoptosis associated with development. However, high levels of dRYBP expression induce apoptosis in all the imaginal discs studied (Figure 1). In transfection experiments, murine and human RYBP/DEDAF proteins have been shown to have the ability to specifically kill mammalian tumor cells while leaving normal cells unaffected $[19,20]$. We have shown that the induction of apoptosis in Drosophila does not require the apoptotic cell to be in the transformed state, indicating that this may be a useful system to study the mechanisms controlling the tumor-specific cell killing. 
Factors involved in the induction of dRYBP-mediated apoptosis

We have shown that dRYBP-induced apoptosis depends on the pro-apoptotic reaper, hid and grim genes (Figure 2C) and that it is inhibited by DIAP1 (Figure 2C). How dRYBP-induced cell death is regulated by these factors is not known. However, we have shown that the promoter region of the reaper gene is bound by the dRYBP protein (Figure $2 \mathrm{~J}$ ) and that dRYBP overexpression activates the expression of a reaper-lac $Z$ construct (Figure 2I). These results suggest that dRYBP regulates the transcription of reaper and that dRYBP-induced apoptosis occurs via the canonical cell death pathways.

We have also shown that dRYBP-induced apoptosis is dependent on the DREDD (Figure 2D) and dFADD proteins (Figure 2E). DREDD is the Drosophila homolog of the mammalian apical caspase-8 [13], while dFADD is the Drosophila homolog of mammalian FADD, an adaptor protein involved in the TNFR signaling complex [14]. Both caspase- 8 and FADD are required for the receptormediated apoptosis in mammals. However, apoptosis induced by the Drosophila TNF homolog EIGER [10] does not require dFADD or DREDD [10]. These results raise the possibility that dRYBP, dFADD and DREDD define a novel Drosophila extrinsic apoptotic pathway. Moreover, studying the mechanisms of dRYBP-mediated cell death in Drosophila might help to define the cell death functions of dFADD and DREDD as well as to better characterize the TNFR apoptotic pathways.

Finally, our results show that dRYBP-induced apoptosis requires the function of trithorax and that TRITHORAX itself is able to induce apoptosis. These findings connect the mechanisms of epigenetic regulation of gene expression with the mechanisms of apoptosis.

Are $d R Y B P$ and TRX involved in the epigenetic regulation of stress-induced apoptosis?

Several observations lead us to propose that dRYBP might be involved in apoptosis associated with cellular stress response. First, we have found no evidence indicating dRYBP involvement in developmental apoptosis. Second, loss of function of the $d R Y B P$ gene results in phenotypes which are highly variable both in penetrance and in expressivity [17]. Third, high levels of RYBP/ DEDAF have been found in a number of different cancers, including T-cell and Hodgkin's lymphomas, pituitary adenomas and oligodendrogliomas [30]. Fourth, it has been shown that the ability of v-Fos transformed cells to invade other tissues is dependent upon repression of RYBP expression. Moreover, re-expression of RYBP in Fos-transformed cells is sufficient to strongly inhibit invasion [31]. Finally, it has been shown in transfection experiments that high levels of RYBP/DEDAF induce tumor cell-specific killing. Therefore, either by induction of apoptosis or by inhibition of invasion, the RYBP protein appears to be able to detect aberrant cell behavior and initiate apoptotic pathways.

We show that high levels of TRX induce apoptosis and, furthermore, that dRYBP-induced apoptosis requires the function of trithorax. These results indicate that the TRX protein might be involved in control of apoptosis and suggest that dRYBP and TRITHORAX could function in the regulation of stress-induced apoptosis.

In our model (Figure 4), under normal conditions of development (Figure 4, left panel), the function of the dRYBP protein remains latent and developmental apoptosis takes place through the canonical pathways of apoptosis proceeding through the activation of the effector caspases followed by cell death. Under conditions (Figure 4 , right panel) where high levels of dRYBP are induced - and similar to conditions in tumor cells where high levels of RYBP have been found [30] - the reaper gene is transcriptionally activated via the dRYBP-dependent recruitment of trxG/PcG complexes. Critical to our model is the establishment of the correct balance between PcG and trxG proteins so that appropriate control of apoptotic gene expression is maintained. This model is consistent with the observed requirement of TRX protein for dRYBP-induced apoptosis (Figure 3B). Although a high level of TRITHORAX can itself activate apoptosis, we propose that the activation of transcription of the apoptotic genes, mediated by the TRX protein, is faster and more accurate when dRYBP is mediating and balancing the recruitment of the $\mathrm{PcG}$ and trxG proteins.

We have shown that dRYBP-induced apoptosis requires the function of DREDD and dFADD (Figure 2D and $2 \mathrm{E}$ ). In mammals the FADD and procaspase- 8 proteins are required for the apoptotic response mediated by the TNF/TNFR complex. This is not the case in Drosophila as the fly TNF/TNFR homologs EIGER/WENGEN do not require dFADD and DREDD to trigger apoptosis. Moreover, in mammals the RYBP/DEDAF protein mediates the interaction between pro-caspase- 8 and FADD to activate caspase- 8 . This leads us to propose that an additional function of the dRYBP protein is to mediate the interaction between AFADD and DREDD to activate the caspase triggering in the apoptotic pathway. dRYBP and TRITHORAX functions may define a novel apoptotic induction pathway that responds to environmental-stress and mutant-stress signals and that requires dFADD and DREDD.

dRYBP-induced apoptosis does not take place in the absence of pro-apoptotic genes reaper, hind and grim (Figure 2B) or in the absence of DREDD/dFADD (Figure 

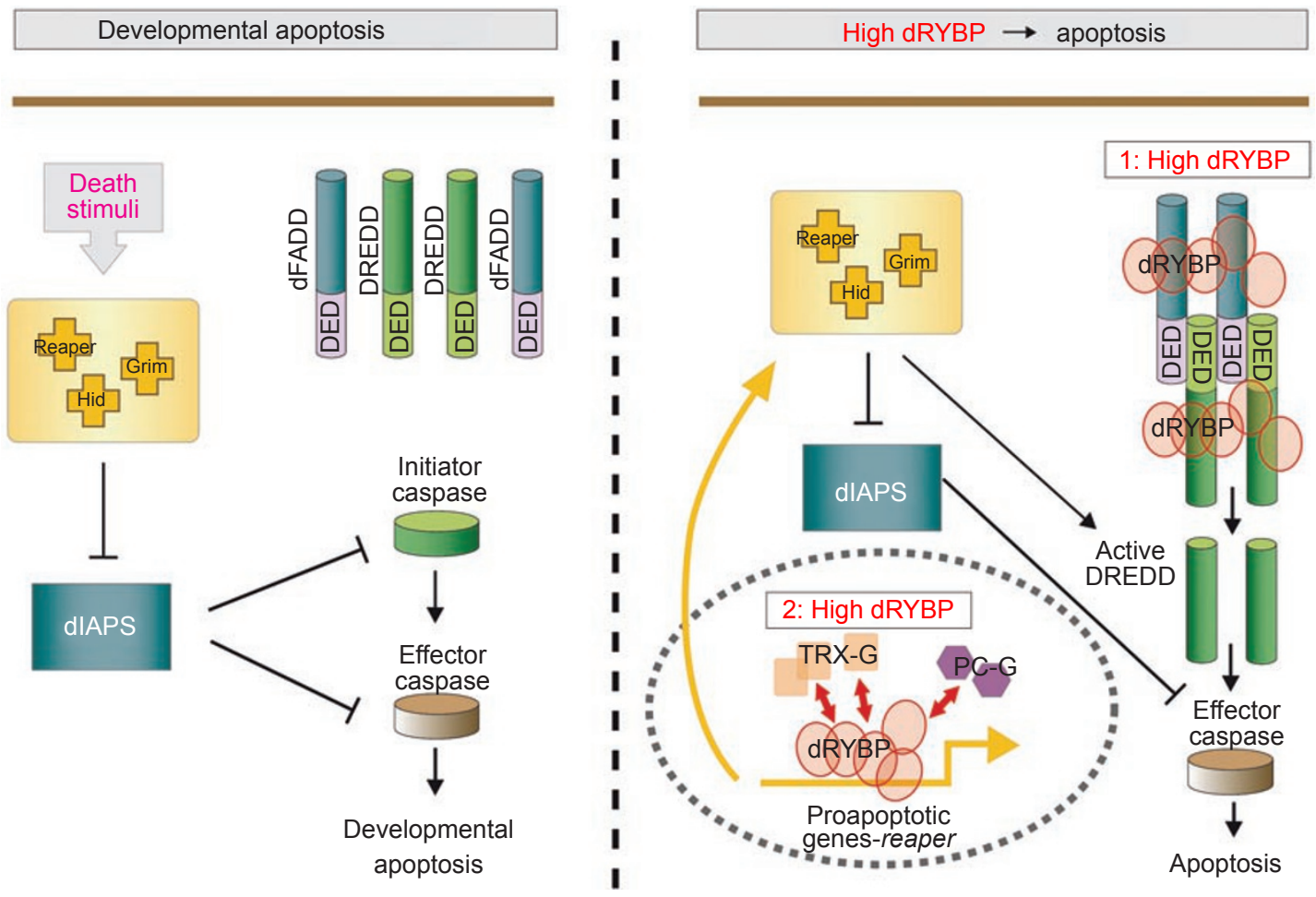

Figure 4 Role of dRYBP and TRX in the epigenetic regulation of apoptosis. Hypothetical model. Developmental apoptosis takes place through the activation, under death stimuli, of the pro-apoptotic genes, which removes the inhibitory action of the DIAP proteins, which in turn allows the initiator and the effector caspases to be activated and induce apoptosis. During developmental apoptosis the function of the dRYBP protein is not required and remains latent. The function of neither dFADD nor the initiator caspase DREDD is required in this process. We hypothesize that the levels of the dRYBP protein are increased, perhaps in response to cellular stress (see text), resulting in the induction of apoptosis. (1) High levels of dRYBP cause the aggregation of the dFADD and the DREDD proteins, thus promoting the activation of DREDD and triggering apoptosis. (2) High levels of $\mathrm{dRYBP}$ activate the expression of the pro-apoptotic gene reaper through the recruitment of the TRX protein, resulting in the induction of apoptosis.

$2 \mathrm{E}$ and $2 \mathrm{D}$ ). It has been previously shown that overexpression of REAPER and overexpression of HID require the presence of $d r e d d$ to induce apoptosis. Thus, DREDD is an effector of REAPER and HID signaling pathways [13]. Moreover, expression of Reaper, Grim and Hid was found to trigger the processing of the DREDD protein precursor [13]. These results support our model and explain why induction of apoptosis by dRYBP is abolished in the absence of either the pro-apoptotic proteins or the adaptor dFADD protein.

How the activation of reaper expression and the activation of DREDD processing function in a pathway induced upon dRYBP overexpression is not known. Nor is it known what stress signals would lead to increased levels of dRYBP and thereby promote apoptosis. We have attempted to identify treatments which would result in increased levels of dRYBP expression in the imaginal discs. None of the treatments investigated - X-ray irradiation, overexpression of EIGER [10], overexpression of HEMIPTEROUS [32], overexpression of REAPER [33], overexpression of TRITHORAX or overexpression of p53 [34] - resulted in increased expression of dRYBP (data not shown). The study of factors controlling dRYBP expression might help to clarify the role of the mammalian RYBP gene in cancer development. Furthermore, elucidating the mechanisms of dRYBP-dependent cell killing and the role of epigenetic regulation by TRX might provide a path to the development of new cancer therapies.

\section{Materials and Methods}

\section{Drosophila strains and handling}

Flies used include stocks containing mutations $P c^{3}, S c e^{l}, t r x^{E 2}$ and $D f(1){ }^{{ }^{667 c 23}}\left(y^{-}, w^{-}\right)$, dredd ${ }^{D 44}, D f(3 L) H 99$ (a deletion that eliminates the pro-apoptotic genes reaper, grim and hid), and they are all described in FlyBase (http://flybase.org). Additionally, the null mutation $d R Y B P^{l}[17,35]$ was used; the stock $d R Y B P^{l} / C y O$ $G F P$ was used to select homozygous $d R Y B P^{l}$ embryos and larvae. 
Transgenic flies containing either puckered-lac ${ }^{E 69}$ (puc-lacZ) [29] or reaper-4kb-lacZ (rpr-lacZ) [36] were used to detect the expression of puckered and reaper, respectively. For overexpression experiments, the GAL4/UAS system was used [37] at $25^{\circ} \mathrm{C}$ and $29^{\circ} \mathrm{C}$ with the following lines (described in Calleja et al. [38]): scalloped-Gal4 (sd-Gal4), nubbin-Gal4 (nub-Gal4), apterus-Gal4 (ap-Gal4), distal less-Gal4 (dll-Gal4, MD23 and EM212) and 248Gal4. Finally, transgenic lines containing UAS-RNAi ${ }_{\text {dRYBP }}[18]$, UAS-RNAi $_{\text {FADD }}{ }^{\text {M8 }}$ [39], UAS-DIAP1 [40], UAS-EIGER [10], UAS-p35 [23], UAS-Dmp53 [34], UAS-TRX (P-EP-TRX, gift of Isabel Guerrero), UAS-SCE [41], UAS-RYBP murine and UASPC (both constructed in this work), UAS-dRYBP [18] and UASdRYBP- $\triangle \mathrm{ZF}$ [17] were used. For imaginal disc irradiation, eggs were collected during a 24-h laying period, allowed to develop until the formation of second instar larvae and then irradiated. After $24 \mathrm{~h}$, the larvae were collected for dissection and immunostaining. Irradiation was performed using a Philips X-ray instrument at a dose of 1 500R.

\section{Staining procedures}

Imaginal discs were dissected in PBS and fixed with 4\% paraformaldehyde in PBS for $30 \mathrm{~min}$ at room temperature. After blocking in a PBT solution containing $1 \%$ bovine serum albumin (BSA) and $0.3 \%$ Triton $\mathrm{X}-100$ for $30 \mathrm{~min}$, discs were incubated overnight at $4{ }^{\circ} \mathrm{C}$ with the primary antibodies. Following three 10 -min washes with PBT (PBS and $0.3 \%$ Triton X-100), they were incubated in the dark with the appropriate fluorescently labelled secondary antibody for $1 \mathrm{~h}$ at room temperature, washed in PBT and mounted in Vectashield (Vector Laboratories). The primary antibodies used were rabbit anti-dRYBP (1:100) [18], rabbit anti-C3 (1:200) (Cell Signalling Technologies) raised against the cleaved form of human caspase-3, which has been shown to cross-react with cleaved Drosophila caspase-3 [22], rabbit anti- $\beta$-Gal (1:200) (Promega), mouse anti- $\beta-$ Gal $(1: 200)$ (Promega), mouse anti-UBX [24] and rabbit anti-phospho-Histone H3 (1:100) (Ser10) (Upstate). Images were generated using either a MicroRadiance (Bio-Rad) or an LSM510 META (Zeiss) confocal microscope, and subsequently processed using Adobe Photoshop.

TUNEL staining was performed using imaginal discs dissected, fixed and incubated with the primary and secondary antibodies as described above for immunostaining. After incubation with the secondary antibody, they were incubated at $65{ }^{\circ} \mathrm{C}$ with NC-T solution $(495 \mu \mathrm{l}$ of sodium citrate $100 \mathrm{mM}, 5 \mu \mathrm{l}$ of Triton X-100 10\%) for $30 \mathrm{~min}$, washed three times for $10 \mathrm{~min}$ in PBT, incubated twice with $100 \mu \mathrm{l}$ of Dilution buffer (Roche) for $5 \mathrm{~min}$ and treated with $50 \mu \mathrm{l}$ of labelling solution (in situ cell death detection TMR Red kit, Roche) for $30 \mathrm{~min}$ at $37^{\circ} \mathrm{C}$. Next, $5 \mu$ of terminal transferase (Roche) was added and the reaction was allowed to proceed for 2 $\mathrm{h}$ at $37^{\circ} \mathrm{C}$, stopped with PBT, washed three times with PBT and the discs were mounted in Vectashield (Vector Laboratories).

BrdU (bromodeoxyuridine) staining was performed using imaginal discs dissected in cold PBS, incubated in $0.01 \mathrm{mM}$ BrdU for $15 \mathrm{~min}$ at $37{ }^{\circ} \mathrm{C}$, washed three times with PBS, fixed for $2 \mathrm{~min}$ with Carnoy (3:1-ethanol:acetic acid) and washed four times for $5 \mathrm{~min}$ in PBS. Discs were then treated with $2 \mathrm{M} \mathrm{HCl}$ for $10 \mathrm{~min}$, washed three times with PBS for $10 \mathrm{~min}$ and incubated with the primary antibody at $4{ }^{\circ} \mathrm{C}$ (BrdU labelling and detection Kit IRoche). The anti-BrdU antibody staining was performed according to the protocol of the supplier.
Acridine Orange staining was performed following the procedure of Abrams [42]. Embryos were dechorionated with bleach, rinsed with water and incubated in $500 \mu \mathrm{l}$ of heptane and $500 \mu \mathrm{l}$ of $5 \mathrm{mg} / \mathrm{ml}$ of Acridine Orange (Sigma). After $5 \mathrm{~min}$ of shaking, embryos were removed from the interface and mounted in Halocarbon oil (Merck).

\section{Expression constructs}

Procedures for the cloning and generation of transgenic flies containing the $\mathrm{p}[\mathrm{UAS}-\mathrm{dRYBP}]$ and $\mathrm{p}[\mathrm{UAS}-\mathrm{dRYBP}-\Delta \mathrm{ZF}]$ proteins have been described previously [17]. The p[UAS-RYBPmurine] construct was generated by cloning the NotI/XbaI fragment from the murine RYBP cDNA (accession number AF101779) [43] into the p[UAST] vector [44]. Likewise, p[UAS-PC] was generated by cloning the PC cDNA (NM 079475) EcoRI fragment into the $\mathrm{p}$ [UAST] vector [44]. Transgenic flies were obtained by standard procedures using $D f(1)^{{ }^{w 67 c 23}}$ flies $\left(y^{-}, w^{-}\right)$as hosts.

\section{Chromatin immunoprecipitation}

ChIP assays using whole embryos were performed as described previously [45]. Chromatin was immunoprecipitated using a 1:20 dilution of the anti-dRYBP antibody [18]. For quantitative ChIP, following immunoprecipitation and DNA purification, enrichment for specific DNA fragments was analyzed by real-time PCR, using the Roche Light Cycler instrument and accessories as described in Negre et al. [45]. Data are expressed as the percentage of input chromatin precipitated for each region examined. The mean values and the standard deviations of two independent ChIP experiments are shown. As a negative control the robo3 (roundabout homolog 3) (http://flybase.org) gene region was included in the RT-PCR experiment. The following primers were used. For reaper CDS: rpr CDS 5'-AGG CGA CTC TGT TGC GGG AG-3' (1oc. 3L: 18391288-18391307; Flybase release 5.10) and rpr CDS 5'-TGC GAT GGC TTG CGA TAT TTG-3' (loc. 3L: 18391142-18391162; Flybase release 5.10). For reaper promoter: rpr promoter 5'-AAA AAC ACG CTT GGC AAC AG-3' (loc. 3L: 18391768-18391787; Flybase release 5.10) and rpr promoter 5'-GCT ATT TAT ACC TGG TTC TCT CAC G-3' (loc. 3L: 18391573-18391597; Flybase release 5.10). For robo3: 5'-ATA GCC TCA ACA CTG AGG AAG G-3' (loc. 2L: 1285910-1285931; Flybase release 5.10) and 3'GTA GGG TTT GAT TAA CCG GAC C-5' (loc. 2L: 12860831286104; Flybase release 5.10). PCR reactions were performed following Comet et al. [46].

\section{Acknowledgments}

We are grateful to Keith Harshman (CIG, University of Lausanne, Switzerland), our colleagues Ernesto Sánchez-Herrero, Francisco Martin, Ricardo Aparicio and members of our group for discussions and for critically reading the manuscript. We especially thank Bernd Schuettengruber (Institute of Human Genetics, Montpellier, France) and members of the laboratory of Giacomo Cavalli for their help with the ChIP and qPCR experiments. We thank our colleague Isabel Guerrero for her generous gift of the UAS-TRX transgenic flies, Bruno Lemaitre (EPFL, Lausanne, Switzerland) for the dredd mutant alleles, and the Bloominton Stock Center, the Hibridoma Bank and the Madrid Drosophila Community for reagents and discussions. We thank our colleague Rocio Simon for help with some of the experiments and colleague José Belio for 
help with Figure 4. This work was supported by grants from Dirección General de Investigación Científica y Técnica (BFU-200502319), the Fundación Investigación Médica Mutua Madrileña (FMM-2006), the Consolider-Ingenio 2010 program of the Ministerio de Ciencia e Innovación (CSD-2007-00008) to AB and by an institutional grant to the Centro de Biología Molecular from the Fundación Ramón Areces.

\section{References}

1 Jacobson MD, Weil M, Raff MC. Programmed cell death in animal development. Cell 1997; 88:347-354.

2 Twomey C, McCarthy JV. Pathways of apoptosis and importance in development. J Cell Mol Med 2005; 9:345-359.

3 Evan G, Littlewood T. A matter of life and cell death. Science 1998; 281:1317-1322.

4 Green DR, Evan GI. A matter of life and death. Cancer Cell 2002; 1:19-30.

5 Degterev A, Boyce M, Yuan J. A decade of caspases. Oncogene 2003; 22:8543-8567.

6 Domingos PM, Steller H. Pathways regulating apoptosis during patterning and development. Curr Opin Genet Dev 2007; 17:294-299.

7 Reed JC. Dysregulation of apoptosis in cancer. J Clin Oncol 1999; 17:2941-2953.

8 Ashkenazi A, Dixit VM. Apoptosis control by death and decoy receptors. Curr Opin Cell Biol 1999; 11:255-260.

9 Igaki T, Kanda H, Yamamoto-Goto Y, et al. Eiger, a TNF superfamily ligand that triggers the Drosophila JNK pathway. EMBO J 2002; 21:3009-3018.

10 Moreno E, Yan M, Basler K. Evolution of TNF signaling mechanisms: JNK-dependent apoptosis triggered by Eiger, the Drosophila homolog of the TNF superfamily. Curr Biol 2002; 12:1263-1268.

11 Grech A, Quinn R, Srinivasan D, Badoux X, Brink R. Complete structural characterisation of the mammalian and Drosophila TRAF genes: implications for TRAF evolution and the role of RING finger splice variants. Mol Immunol 2000; 37:721-734.

12 Liu H, Su YC, Becker E, Treisman J, Skolnik EY. A Drosophila TNF-receptor-associated factor (TRAF) binds the ste20 kinase Misshapen and activates Jun kinase. Curr Biol 1999; 9:101-104.

13 Chen P, Rodriguez A, Erskine R, Thach T, Abrams JM. Dredd, a novel effector of the apoptosis activators reaper, grim, and hid in Drosophila. Dev Biol 1998; 201:202-216.

$14 \mathrm{Hu}$ S, Yang X. dFADD, a novel death domain-containing adapter protein for the Drosophila caspase DREDD. J Biol Chem 2000; 275:30761-30764.

15 Meyer HH, Wang Y, Warren G. Direct binding of ubiquitin conjugates by the mammalian p97 adaptor complexes, p47 and Ufd1-Npl4. EMBO J 2002; 21:5645-5652.

16 Arrigoni R, Alam SL, Wamstad JA, et al. The Polycombassociated protein Rybp is a ubiquitin binding protein. FEBS Lett 2006; 580:6233-6241.

17 Gonzalez I, Aparicio R, Busturia A. Functional characterization of the dRYBP gene in Drosophila. Genetics 2008; 179:1373-1388.

18 Bejarano F, Gonzalez I, Vidal M, Busturia A. The Drosophila
RYBP gene functions as a Polycomb-dependent transcriptional repressor. Mech Dev 2005; 122:1118-1129.

19 Novak RL, Phillips AC. Adenoviral-mediated Rybp expression promotes tumor cell-specific apoptosis. Cancer Gene Ther 2008; 15:713-722.

20 Danen-van Oorschot AA, Voskamp P, Seelen MC, et al. Human death effector domain-associated factor interacts with the viral apoptosis agonist Apoptin and exerts tumor-preferential cell killing. Cell Death Differ 2004; 11:564-573.

21 Mazo AM, Huang DH, Mozer BA, Dawid IB. The trithorax gene, a trans-acting regulator of the bithorax complex in Drosophila, encodes a protein with zinc-binding domains. Proc Natl Acad Sci USA 1990; 87:2112-2116.

22 Yu SY, Yoo SJ, Yang L, et al. A pathway of signals regulating effector and initiator caspases in the developing Drosophila eye. Development 2002; 129:3269-3278.

23 Hay BA, Wolff T, Rubin GM. Expression of baculovirus P35 prevents cell death in Drosophila. Development 1994; 120:2121-2129.

24 White RA, Wilcox M. Distribution of Ultrabithorax proteins in Drosophila. EMBO J 1985; 8:2035-2043.

25 Goyal L, McCall K, Agapite J, Hartwieg E, Steller H. Induction of apoptosis by Drosophila reaper, hid and grim through inhibition of IAP function. EMBO J 2000; 19:589-597.

26 Yoo SJ, Huh JR, Muro I, et al. Hid, Rpr and Grim negatively regulate DIAP1 levels through distinct mechanisms. Nat Cell Biol 2002; 4:416-424.

27 White K, Grether ME, Abrams JM, Young L, Farrell K, Steller H. Genetic control of programmed cell death in Drosophila. Science 1994; 264:677-683.

28 Hengartner MO. The biochemistry of apoptosis. Nature 2000; 407:770-776.

29 Martin-Blanco E, Gampel A, Ring J, et al. Puckered encodes a phosphatase that mediates a feedback loop regulating JNK activity during dorsal closure in Drosophila. Genes Dev 1998; 12:557-570.

30 Sanchez-Beato M, Sanchez E, Garcia JF, et al. Abnormal PcG protein expression in Hodgkin's lymphoma. Relation with E2F6 and NFkappaB transcription factors. J Pathol 2004; 204:528-537.

31 McGarry LC, Winnie JN, Ozanne BW. Invasion of v-Fos(FBR)transformed cells is dependent upon histone deacetylase activity and suppression of histone deacetylase regulated genes. Oncogene 2004; 23:5284-5292.

32 Adachi-Yamada T, Fujimura-Kamada K, Nishida Y, Matsumoto K. Distortion of proximodistal information causes JNK-dependent apoptosis in Drosophila wing. Nature 1999; 400:166169.

33 Wing JP, Schwartz LM, Nambu JR. The RHG motifs of Drosophila Reaper and Grim are important for their distinct cell death-inducing abilities. Mech Dev 2001; 102:193-203.

34 Peters M, DeLuca C, Hirao A, et al. Chk2 regulates irradiation-induced, p53-mediated apoptosis in Drosophila. Proc Natl Acad Sci USA 2002; 99:11305-11310.

35 Bellen HJ, Levis RW, Liao G, et al. The BDGP gene disruption project: single transposon insertions associated with $40 \%$ of Drosophila genes. Genetics 2004; 167:761-781.

36 Jiang C, Lamblin AF, Steller H, Thummel CS. A steroidtriggered transcriptional hierarchy controls salivary gland 
cell death during Drosophila metamorphosis. Mol Cell 2000; 5:445-455.

37 Brand AH, Manoukian AS, Perrimon N. Ectopic expression in Drosophila. Methods Cell Biol 1994; 44:635-654.

38 Calleja M, Moreno E, Pelaz S, Morata G. Visualization of gene expression in living adult Drosophila. Science 1996; 274:252255.

39 Naitza S, Rosse C, Kappler C, et al. The Drosophila immune defense against gram-negative infection requires the death protein dFADD. Immunity 2002; 17:575-581.

40 Hay BA, Wassarman DA, Rubin GM. Drosophila homologs of baculovirus inhibitor of apoptosis proteins function to block cell death. Cell 1995; 83:1253-1262.

41 Gorfinkiel N, Fanti L, Melgar T, et al. The Drosophila Polycomb group gene Sex combs extra encodes the ortholog of mammalian Ring1 proteins. Mech Dev 2004; 121:449-462.
42 Abrams JM, White K, Fessler LI, Steller H. Programmed cell death during Drosophila embryogenesis. Development 1993; 117:29-43.

43 Garcia E, Marcos-Gutierrez C, del Mar Lorente M, Moreno JC, Vidal M. RYBP, a new repressor protein that interacts with components of the mammalian Polycomb complex, and with the transcription factor YY1. EMBO J 1999; 18:3404-3418.

44 Brand AH, Perrimon N. Targeted gene expression as a means of altering cell fates and generating dominant phenotypes. Development 1993 ; 118:401-415.

45 Negre N, Lavrov S, Hennetin J, Bellis M, Cavalli G. Mapping the distribution of chromatin proteins by ChIP on chip. Methods Enzymol 2006; 410:316-341.

46 Comet I, Savitskaya E, Schuettengruber B, et al. PRE-mediated bypass of two $\mathrm{Su}(\mathrm{Hw})$ insulators targets $\mathrm{PcG}$ proteins to a downstream promoter. Dev Cell 2006; 11:117-124. 